\title{
Detection of Knee Joint Disorders using SVM Classifier
}

Alphonsa Salu S. J*, Jeraldin Auxillia D.

Department of Electronics and Communication Engineering, St. Xaviers Catholic College of Engineering, Chunkankadai, Tamil Nadu, India

\section{ABSTRACT}

\section{Article Info}

Volume 8, Issue 5

Page Number : 261-271

\section{Publication Issue}

September-October-2021

\section{Article History}

Accepted : 01 Sep 2021

Published : 05 Sep 2021
A non-invasive technique using knee joint vibroarthographic (VAG) signals can be used for the early diagnosis of knee joint disorders. Among the algorithms devised for the detection of knee joint disorders using VAG signals, algorithms based on entropy measures can provide better performance. In this work, the VAG signal is preprocessed using wavelet decomposition into sub band signals. Features of the decomposed sub bands such as approximate entropy, sample entropy \& wavelet energy are extracted as a quantified measure of complexity of the signal. A feature selection based on Principal Component Analysis (PCA) is performed in order to select the significant features. The extracted features are then used for classification of VAG signal into normal and abnormal VAG using support vector machine. It is observed that the classifier provides a better accuracy with feature selection using principal component analysis. And the results show that the classifier was able to classify the signal with an accuracy of $82.6 \%$, error rate of 0.174 , sensitivity of 1.0 and specificity of 0.888 .

Keywords : Vibroarthrography, Wavelet decomposition, Feature extraction, Principal Component Analysis, support Vector Machine.

\section{INTRODUCTION}

Vibroarthography (VAG) can be used as a noninvasive diagnostic tool to detect articular cartilage degeneration. The knee joint disorders due to articular cartilage degeneration could be easily found out using medical imaging techniques like Magnetic Resonance Imaging (MRI) and Computed Tomography (CT). These techniques can capture only gross cartilage defects but fail to provide an early diagnosis of articular degeneration. In conventional arthroscopy where the cartilage surface is inspected with a fiber optic cable would not be feasible for a patient's repeated or periodic checkups. In contrast, the vibration or auditory signals known as Vibroarthographic (VAG) signal emitted from the mid-patella during the active movements of legs such as the flexion and extension enables the early and better diagnosis of knee joint disorders. [1].

In a large number of published research works the VAG signal is characterized as non-stationary, aperiodic and non-linear signal, just like the other 
natural signals due to the fact that the quality of joint surfaces coming in contact may not be the same from one angular position to another due to the articulation of the joint [2]. The VAG signal is said to be a multicomponent signal because the signal from a single source (within the knee cartilage) can propagate through different channels of tissue to the mid-patella position, giving rise to multiple energy components at different frequencies at a time. From these analogies it is understood that VAG signals cannot be easily analysed by common signal processing techniques such as the Fourier transform or autoregressive modelling. In this work, Saif Nalband et al [2] describes a method for analysis of VAG signals using Ensemble Empirical Mode Decomposition (EEMD). The features such as Tsallis entropy, permutation entropy and spectral entropy are used for classifying the signals with an accuracy of $86.52 \%$.

From the literature survey it is known that the EEG signal which has the similar properties of VAG signal is decomposed using Discrete Wavelet Transform (DWT) and the features are extracted which provides a better classification [4,5]. Elif Derya Ubeyli [4] proposed the decomposition of EEG signals into timefrequency representations using DWT and achieved a classification accuracy of $94.83 \%$ using combined neural network. Mingyang Li et al. [5] also proposed a method of decomposing the EEG signals using DWT along with the envelope analysis (EA) technique and an effective network model called Neural Network Ensemble (NNE) is used for classifying the signals with an accuracy of $98.78 \%$.

Kaizhi Liu et al. [6[ and Yungfeng Wu et al. [7] describes a classification of VAG signals using features like Form Factor (FF) and Variance of Mean Squared values (VMS). Yunfeng Wu et al. [8] describes the analysis of VAG signals using features like Symbolic Entropy (SyEn), Approximate Entropy (ApEn), Fuzzy Entropy (FuzzyEn), mean, SD and RMS values of the envelope amplitude. The results showed that Support Vector Machine (SVM) based on entropy features can provide superior classification accuracy than Quadratic Discriminant Analysis (QDA) and Generalised Logistic Regression Analysis (GLRA) with an accuracy of $83.56 \%$.

The statistical parameters such as Form Factor (FF), Kurtosis (K), Skewness (S) and Entropy $(\mathrm{H})$ are extracted as features to classify VAG signals using linear Strict 2-surface Proximal (S2SP) classifier and non-linear S2SP classifier. In this work Tingting $\mathrm{Mu}$ et al. [9] was able to achieve a classification accuracy of $74.2 \%$ with linear S2SP classifier along with genetic algorithm (GA) and an accuracy of $91 \%$ with non-linear S2SP classifier along with GA. For a better classification accuracy it is necessary to choose the dominant features using feature selection algorithms. In the previous research works, Ahmed Taher Azar et al [10] used feature selection algorithms such as Genetic Algorithm (GA), Principal Component Analysis (PCA), Relief F, Fisher, Sequential Forward floating Search (SFFS) and Sequential Backward Floating Search (SBFS). It was observed that the classification involving the feature selection method of GA achieved a classification accuracy of 92.2\%. In another work, Sridhar Krishnan et al. [17] describes the time frequency analysis of VAG signals with minimum cross-entropy optimization and matching pursuit decomposition. It shows that the selected features of the VAG signal could provide an accuracy of $77.5 \%$.

The methods of feature selection like sequential forward selection and sequential backward selection were found to be slow in order to check the dominant feature subset for prediction error. Whereas, the method of Principal Component Analysis (PCA) can be applied to determine the best set of features which can provide more accuracy to classify the signals [21]. It is understood that PCA has been successfully used to reduce the dimension of features of biomedical 
signals without rejecting the important features providing a sensitivity of $98 \%$. From this it can be made clear that the data of high dimension and irrelevant features reduces the accuracy.

Karthikeyan Umapathy and Sridhar Krishnan [22] proposed a method of classifying VAG signals using Linear Discriminant Bases algorithm with an accuracy of 76.4\%. Keo-Sik Kim et al. [24] proposed a method of classifying the VAG signals into 2 classes of normal and abnormal signals using Back Propogation Neural Network (BPNN) with an accuracy of 92.3\%. Shanshan Yang et al [25] describes a method of classifying normal and pathological signal groups using Bayesian classifier and Least Square Support Vector Machine (LS-SVM). In this work Bayesian classifier made better classification compared to LSSVM with an accuracy of $88 \%$.

The aim of this paper is to improve the classification accuracy of features obtained from wavelet decomposition using Principal Component Analysis (PCA) method of feature selection and to evaluate the performance of the proposed method using Support Vector Machine (SVM). The features such as Approximate Entropy (ApEn), Sample Entropy (SampEn) and Wavelet energy are extracted and only the selected features using PCA are provided to classifiers. Also the performance parameters such as accuracy, error rate, sensitivity and specificity are calculated for each classifiers.

\section{METHODS AND MATERIAL}

\section{A. Data Acquisition System}

The dataset used in this work is from the experiment for data acquisition of VAG signal conducted in the University of Calgary, Canada. The set up required the subject to sit on an adamant surface table with leg dropping freely in air. The subject was asked to perform extension and flexion of the knee joint at an angle of $\left(90^{\circ}-0^{\circ}-90^{\circ}\right)$ degrees. The experiment was conducted for 4 sec/cycle. The signal is pre-filtered (10 Hz-1KHZ) and amplified before sampling at a rate of $2 \mathrm{KHz}$ and digitized with a resolution of 12 bits. The dataset consists of 89 VAG signals in which 51 VAG signals are obtained from 51 healthy subjects having no physiological disorder condition and 38 VAG signals are obtained from 38 subjects suffering from various kinds of knee joint disorders [2].

\section{B. Proposed Work}

The block diagram of the proposed method is as shown in Fig.1

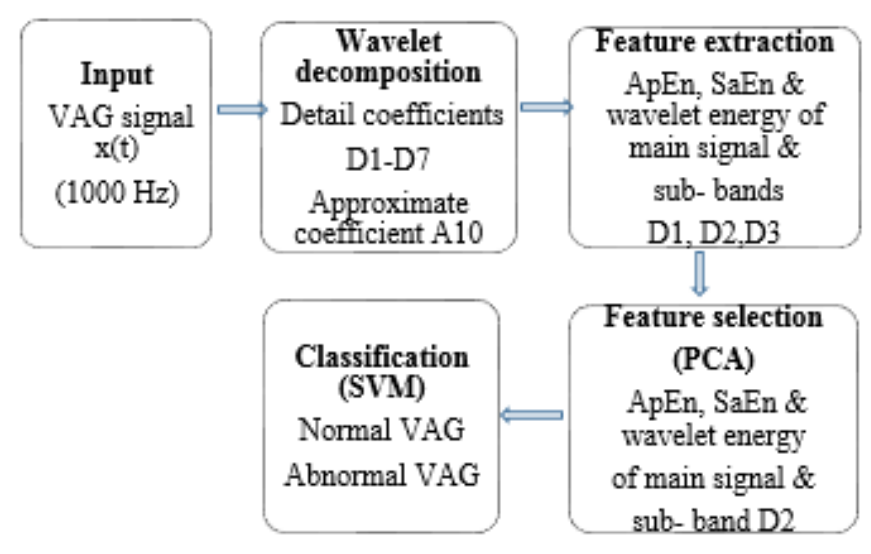

Fig.1 Block diagram of VAG detection system

i) Wavelet Decomposition: Wavelet decomposition involves wavelet transform were the discrete-time sampled signal is passed through filters. In wavelet decomposition input signal is split into a number of sub-bands of different frequencies. It performs successive low pass and high pass filtering of the discrete time domain signal. At each level the high pass signal produces detail information while the low pass filter associated with scaling function produces coarse approximations. In the proposed work wavelet decomposition is performed using Daubechies $4(\mathrm{db} 4)$ as the mother wavelet which is regarded as an efficient wavelet to analyse biomedical signal [3]. The original data sequence $\mathrm{x}(\mathrm{t})$ is taken with $\mathrm{N}$ data points such as $\mathrm{X}[\mathrm{x}(1), \mathrm{x}(2), \ldots, \mathrm{x}(\mathrm{N})]$. The wavelet coefficient 
$\gamma$ of a signal $x(n)$ is the projection of $x(t)$ onto a wavelet (Daubechies,db4-mother wavelet) $\psi(t))$ given by the equation,

$$
\psi_{j, k}(t)=\frac{1}{\sqrt{2^{j}}} \psi\left(\frac{t-k 2^{j}}{2^{j}}\right)
$$

Where $\mathrm{j}$ is the scale parameter, $\mathrm{k}$ is the shift parameter and both are integers. The wavelet coefficient can be given by the equation

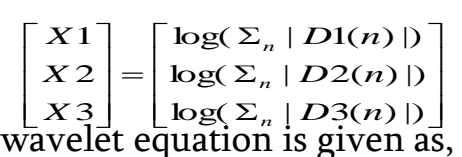

The mother wavelet equation is given as,

$$
\gamma_{j k}=\int_{-\infty}^{\infty} x(t) \frac{1}{\sqrt{2^{j}}} \psi\left(\frac{t-k 2^{j}}{2^{j}}\right) d t
$$

ii) Feature Extraction: The sub-bands obtained after wavelet decomposition are fed as input to the feature extraction process. Features such as Approximate Entropy (ApEn), Sample Entropy (SaEn) and wavelet energy are extracted from the main signal and subband signals D1, D2 and D3.

Approximate entropy is used to quantify the amount of regularity and the unpredictability of fluctuations over time series data. It detects changes in the underlying behaviour and compares the similarity of the samples by pattern length (m) and similarity coefficient (r). Highly irregular time series gives a high ApEn value and a time series with more number of similarity patterns gives a low ApEn value. Mathematically, ApEn is calculated by the equation,

$$
n(x)=\frac{1}{\sqrt{2^{j}}} \mu\left(\frac{-t}{2^{j}}\right)
$$

where, ' $\mathrm{N}$ ' represents the number of data points of the given input signal, ' $m$ ' represents the length of the particular data segment being compared, 'r' represents similarity criterion which is the measure of distance between the elements of the compared data segments, $\varphi^{m}(r)$ is the pattern mean of length $\mathrm{m}$ and $\varphi^{m+1}(r)$ is the pattern mean of length $\mathrm{m}+1$. The pattern mean is calculated by computing the count of similar patterns of length $\mathrm{m}$ and length $\mathrm{m}+1$. Usually $\mathrm{m}$ is chosen to be either one or two and $r$ is chosen between 0.1 and 0.25 times the standard deviation of the original time series as suggested by Pincus [13]. In this work ' $\mathrm{m}$ ' and ' $\mathrm{r}$ ' are chosen as $\mathrm{m}=2$ and $\mathrm{r}=0.3$ respectively.

Sample entropy is a measure of complexity. But it does not include self-similar patterns. It measures the regularity of a physiological signal and is independent of the pattern length. If SampEn value of a dataset is higher than the other for a given pattern length (m) and similarity criterion ( $r$ ) then it remains higher for all the different values of $\mathrm{m}$ and $\mathrm{r}$. Thus, SampEn is relatively consistent and reduces the bias of approximate entropy. In the proposed work $\mathrm{m}=2$ and $r=0.3$ are chosen according to Pincus [13]. High value of SampEn implies that the signal is highly unpredictable and a low SampEn value implies the signal is predictable. Sample entropy is computed using the equation,

$$
\text { SampEn }=\log \left(\frac{A}{B}\right)
$$

where A contains the total number of vector pairs of length $\mathrm{m}+1$ and $\mathrm{B}$ contains total number of vector pairs of length $\mathrm{m}$.

Wavelet energy indicates the percentage of energy corresponding to the approximate and detail coefficients obtained after wavelet decomposition [14]. Wavelet based energy of each of the sub-band signals D1, D2, D3 are computed using the equation,

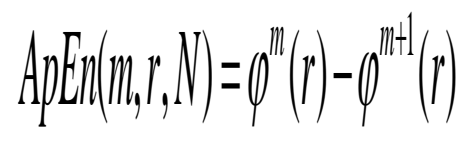

Where $\mathrm{n}$ represents the number of coefficients in each sub-band D1(n) ,n=503; D2(n), n=255; D3(n), $\mathrm{n}=131$.

iii) Feature Selection: In the proposed work, the feature selection is performed using a technique called Principal Component Analysis (PCA). This method uses the correlation between the features in order to identify the most significant features which is taken as the principal component and it is used for the further classification of the signals into normal and abnormal [21]. Consider the data set $\mathrm{X}=\left(\mathrm{x}_{1}, \mathrm{x}_{2}\right.$, $\left.\mathrm{x}_{3}, \ldots, \mathrm{xN}\right)$. The first step to compute the principal 
components is the calculation of the mean of $\mathrm{X}$ using the formula,

$$
\operatorname{Mean}(X)=x^{\prime}=\frac{1}{N} \sum_{i=1}^{N}\left(x_{i}\right)
$$

The next step is to compute the covariance in order to calculate the correlation between features. The covariance is calculated using the formula,

$$
\operatorname{cov}(x)=\frac{1}{N}\left(x_{i}-x_{i}^{\prime}\right)\left(x_{i}-x_{i}^{\prime}\right)^{T}
$$

And finally, the last step is to compute the Eigen vectors and Eigen values of the covariance matrix obtained above [8]. These eigen values are sorted as $\lambda_{1} \geq \lambda_{2} \geq \lambda_{3} \ldots \geq \lambda_{D}$ which gives $Y=\left(y_{1}, y_{2}, y_{3}, \ldots y_{p}\right)$ such that $Y$ is the lower $\mathrm{D}$ dimensional data set with the principal components.

iv) Classification using SVM: In machine learning, support vector machines (SVM's) are supervised learning algorithms that analyze data used for classification and regression analysis. An SVM model

\begin{tabular}{|l|l|l|l|}
\hline & $\begin{array}{l}\text { Predicted } \\
\text { NO }\end{array}$ & $\begin{array}{l}\text { Predicted } \\
\text { YES }\end{array}$ & FP \\
\hline $\begin{array}{l}\text { Actual } \\
\text { NO }\end{array}$ & TN & $\begin{array}{l}\text { Total } \\
\text { predicted } \\
\text { NO }\end{array}$ \\
\hline $\begin{array}{l}\text { Actual } \\
\text { YES }\end{array}$ & FN & TP & $\begin{array}{l}\text { Total } \\
\text { predicted } \\
\text { YES }\end{array}$ \\
\hline $\begin{array}{l}\text { Total } \\
\text { actual } \\
\text { NO }\end{array}$ & $\begin{array}{l}\text { Total } \\
\text { actual } \\
\text { YES }\end{array}$ & \\
\hline
\end{tabular}

is a representation of the examples as points in space, mapped so that the examples of the separate categories are divided by a clear gap that is as wide as possible. New examples are then mapped into the same space and predicted to belong to a category based on which side they fall [23]. Maximum margin hyper plane and margin for an SVM trained with samples from two classes is shown in the Fig.2.

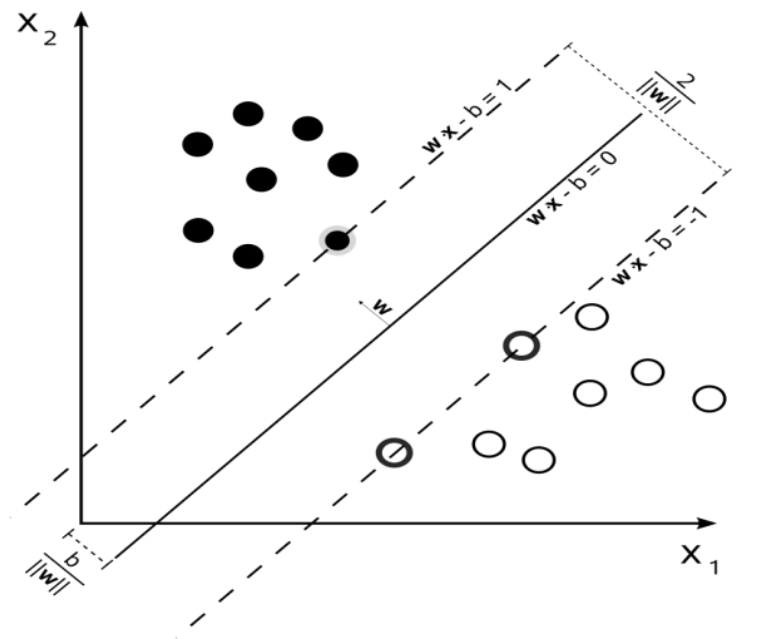

Fig.2 Maximum-margin hyper plane and margins for an SVM trained with samples from two classes

SVM performs classification by finding the optimal hyperplane (OHP). To find OHP, SVM aims to maximize the margin. Distance between data point $\mathrm{x}$ to OHP is given by the equation $\mathrm{D}(\mathrm{x}) /\|\mathrm{w}\|$ where $\mathrm{w}$ is the weight and $\mathrm{D}(\mathrm{x})$ is the discriminant function given by $\mathrm{w}^{\mathrm{T}} \mathrm{x}+\mathrm{b}$.

\section{Performance Measures}

The performance of the proposed method is evaluated by the parameters Accuracy, Error rate, Sensitivity and Specificity obtained by forming the confusion matrix. Diagonal matrix indicates correctly classified states when the output matches the target. The other blocks correspond to misclassification [23]. The general representation of confusion matrix is shown in Fig.3 .

Fig.3 Confusion matrix for a 2-class problem

Where TP is true positive - number of diseased states correctly identified as diseased.

TN is true negative - number of healthy states identified as healthy.

FP is false positive - number of healthy states identified as diseased. 
FN is false negative - number of diseased states identified as healthy.

Accuracy measures the degree of closeness of measurements of a quantity to its actual value. Error rate measures the number of all incorrect predictions to the total number of dataset. Sensitivity refers to the ability of a test to correctly identify those with the disease (true positive rate). Specificity refers to the ability of the test to correctly identify those without disease.

Accuracy $=(\mathrm{TP}+\mathrm{TN}) /$ Total no. of test samples.

Error rate $=(\mathrm{FP}+\mathrm{FN}) /$ Total no. of test samples.

Sensitivity $=$ TN/ACTUAL DISEASED .

Specificity $=$ TP/ACTUAL HEALTHY.

\section{III.RESULTS AND DISCUSSION}

\section{A.Database}

The dataset consists of 89 VAG signals in which 51 VAG signals are obtained from 51 healthy subjects having no physiological disorder condition and 38 VAG signals are obtained from 38 subjects suffering from various kinds of knee joint disorders. In the proposed work a total of 20 signals are taken as input from the above mentioned dataset. Among these 11 are abnormal (with pathological disorders) VAG signals and 9 are normal (without any pathological disorders) VAG signals. The sample input signal is shown in Fig.4 .

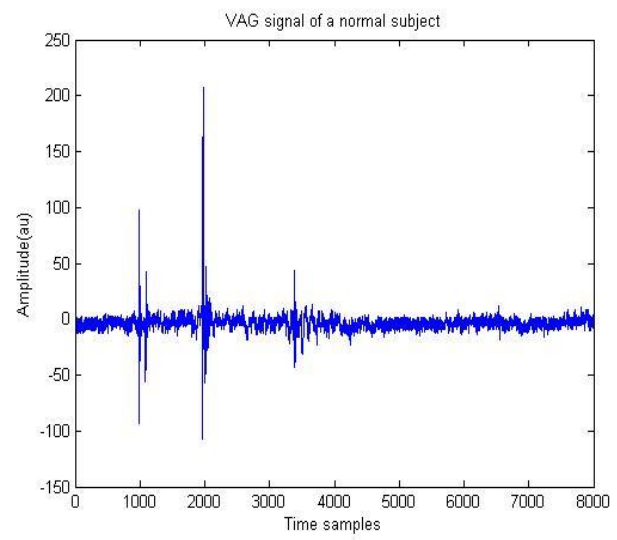

(a)Normal VAG signal

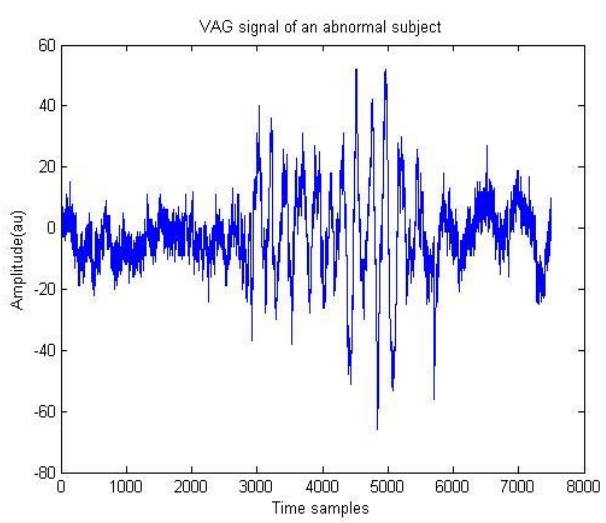

(b) Abnormal VAG signal

Fig.4 Input signal (a) Normal VAG signal (b) Abnormal VAG signal

\section{B. Wavelet Decomposition}

The input signal is pre-processed using wavelet decomposition and split into a number of sub-bands of detail coefficients from D1 to D7 and approximation coefficient A10 as Table 1.

TABLE 1. DECOMPOSITION OF VAG SIGNALS INTO SUB-BAND SIGNALS

\begin{tabular}{|c|c|c|c|}
\hline $\begin{array}{c}\text { Sub- } \\
\text { band }\end{array}$ & $\begin{array}{c}\text { Frequency } \\
(\mathrm{Hz})\end{array}$ & $\begin{array}{c}\text { Sub- } \\
\text { band }\end{array}$ & $\begin{array}{c}\text { Frequency } \\
(\mathrm{Hz})\end{array}$ \\
\hline D1 & $1000-500$ & D5 & $62.5-31.25$ \\
\hline D2 & $500-250$ & D6 & $31.25-15.6$ \\
\hline D3 & $250-125$ & D7 & $15.6-7.81$ \\
\hline D4 & $125-62.5$ & A10 & $1-0$ \\
\hline
\end{tabular}

The sub band signals containing the detail coefficients cd101, cd102, cd105, cd110 of the normal VAG signal are shown in Fig.5. And the sub band signals containing the detail coefficients such as $\mathrm{cd} 1, \mathrm{~cd} 2, \mathrm{~cd} 3$ and cd4 of the abnormal VAG signal are shown in Fig.6. 


\section{Feature Extraction}

The main signal and sub band signals containing the detail coefficients are used for extraction of features such as approximate entropy, sample entropy and wavelet energy. From the sub bands
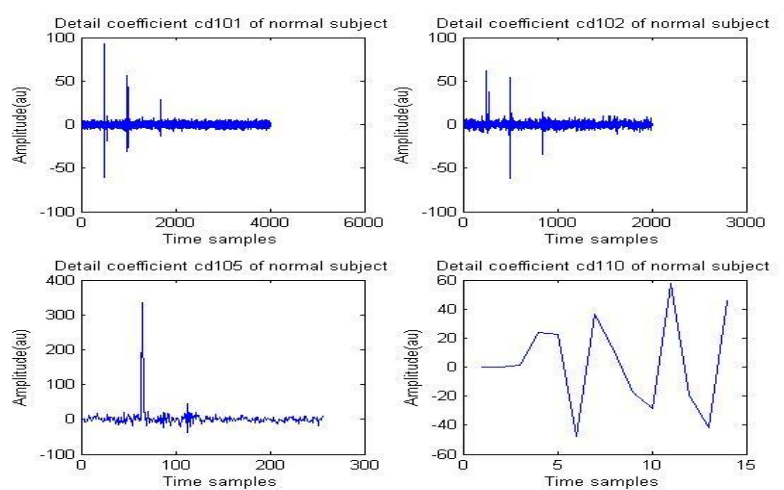

Fig.5 Sub band signals containing detail coefficients of the normal VAG signal
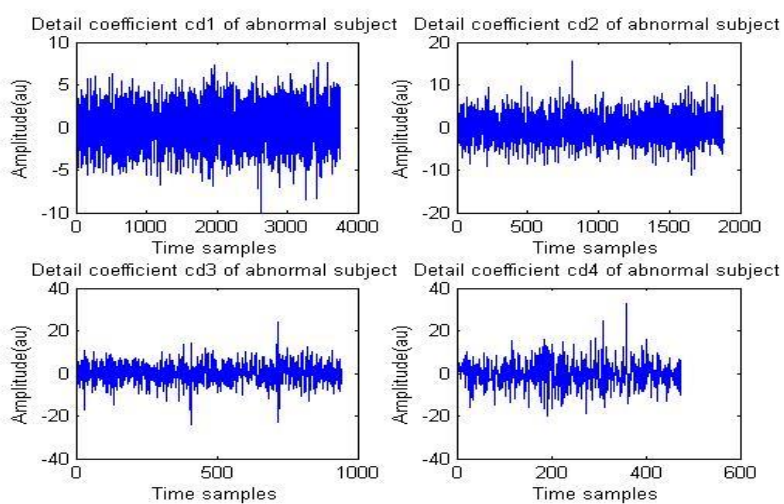

Fig.6 Sub bands containing detail coefficients of the abnormal VAG signal

obtained after wavelet decomposition the sub bands D1, D2 and D3 are chosen for feature extraction. The extracted features are,

(a) Approximate entropy of main signal and sub-bands D1, D2,D3.

(b) Sample entropy of main signal and subbands D1, D2, D3.

(c) Wavelet energy of main signal and subbands D1, D2,D3 .

\section{Feature Selection}

The feature selection is done based on the Principal Component analysis (PCA) test. To verify the discriminability between the features, the PCA test is performed on the features extracted. It is a test of correlation between the features. The p-value returned from the test indicates the variation between the data. Smaller p-value of 0.05 indicates that the feature data belongs to the same group and could be a useful feature in distinguishing the two classes. The results obtained from PCA test for the selected features are shown in Table 2.

TABLE 2. SELECTED FEATURES OBTAINED FROM PCA TEST

\begin{tabular}{|l|l|}
\hline Features & $\mathrm{p}$-value \\
\hline Apen of D2 & 0.4751 \\
\hline Apen of main signal & 0.0367 \\
\hline Saen of D2 & 0.2531 \\
\hline Saen of main signal & 0.1385 \\
\hline Wavelet energy of D2 & 0.1985 \\
\hline Wavelet energy of main signal & 0.4703 \\
\hline
\end{tabular}

\section{E. Classification}

The classification is done in two different ways such as classification without feature selection and with feature selection using Support Vector Machine classifier.

\section{i) Classification without feature selection: The} extracted features are given as input to the support vector machine classifier to classify the signal into two classes. Training is done using 38 sets of data containing all the extracted features. Testing is done using 23 sets of data. Out of 23, the SVM classifier correctly classified 18 sets of data with an accuracy of $78.26 \%$. 
ii) Classification with feature selection: In this classification only the selected features obtained from the principal component analysis test for feature selection are given as input to the support vector machine classifier to classify the signal into two classes. Here, training is done using 6 sets of data containing only the significant features. Testing is done using the same 23 sets of data. Out of 23 , the SVM classifier correctly classified 19 sets of data with an accuracy of $82.6 \%$. From this it is understood that the classification with feature selection provides more accuracy.

\section{F. Performance Analysis}

The performance of the proposed method is evaluated by the parameters Accuracy, Error rate, Sensitivity and Specificity obtained by forming the confusion matrix. The classified output is evaluated using confusion matrix. Diagonal matrix indicates correctly classified states when the output matches the target. The other blocks correspond to misclassification. The confusion matrix obtained for classification without feature selection using SVM classifier is shown in Fig.7.

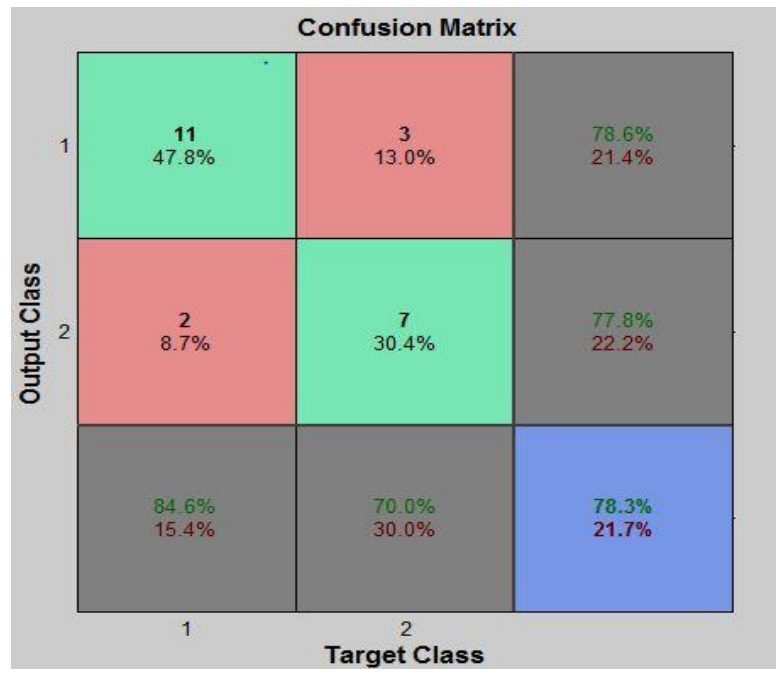

Fig.7 Confusion matrix - Classification without

feature selection using SVM classifier

And the confusion matrix obtained for classification with feature selection using SVM classifier is shown in Fig.8

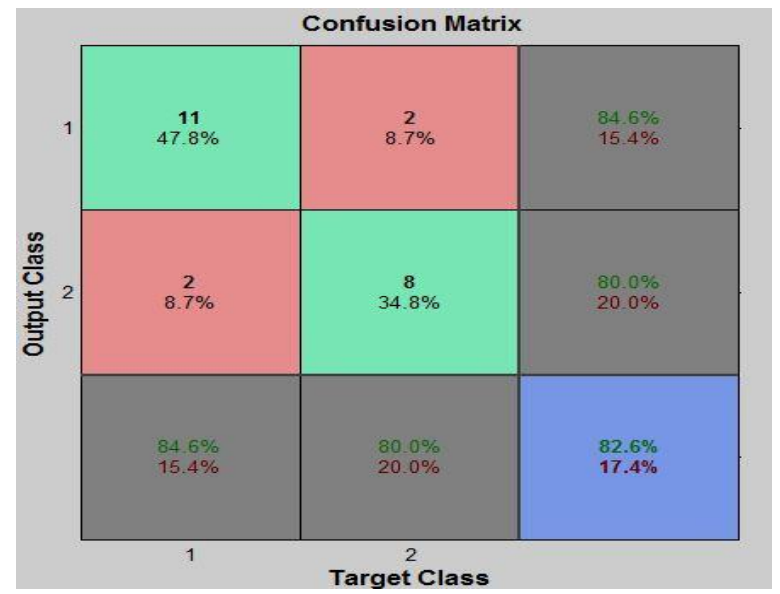

Fig.8 Confusion matrix - Classification with feature selection using SVM

Performance measures that evaluate the performance of the classifiers without using feature selection and classifiers with feature selection is shown in Table 3. Out of a set of 23 features used for testing, the classification without feature selection using SVM classifier shows an accuracy of $78.26 \%$, error rate of 0.217 , sensitivity of 1.0 and specificity of 0.777 . And out of the same set of 23 features used for testing, the classification with feature selection using SVM classifier shows an accuracy of $82.6 \%$, error rate of 0.174 , sensitivity of 1 and specificity of 0.888 .

TABLE 3. PERFORMANCE ANALYSIS OF SVM CLASSIFIER

\begin{tabular}{|l|l|l|l|}
\hline \multicolumn{4}{|c|}{ Classification without feature selection } \\
\hline Accuracy & Error rate & Sensitivity & Specificity \\
\hline $78.3 \%$ & 0.217 & 1.0 & 0.777 \\
\hline \multicolumn{4}{|c|}{ Classification with feature selection } \\
\hline Accuracy & Error rate & Sensitivity & Specificity \\
\hline $82.6 \%$ & 0.174 & 1.0 & 0.888 \\
\hline
\end{tabular}

The results obtained by the proposed method is compared with the previous literature studies using the same set of data as shown in Table 4. 
TABLE 4. COMPARISON WITH THE EXISTING WORK

\begin{tabular}{|l|l|l|l|l|}
\hline Classifier & $\begin{array}{l}\text { Accurac } \\
\mathrm{y}\end{array}$ & $\begin{array}{l}\text { Erro } \\
\mathrm{r} \\
\text { rate }\end{array}$ & $\begin{array}{l}\text { Sensitivit } \\
\mathrm{y}\end{array}$ & $\begin{array}{l}\text { Specificit } \\
\mathrm{y}\end{array}$ \\
\hline $\begin{array}{l}\text { SVM } \\
\text { (with } \\
\text { PCA) }\end{array}$ & $82.6 \%$ & 0.17 & 1.0 & 0.888 \\
\hline $\begin{array}{l}\text { SVM } \\
\text { (Yungfen } \\
\text { g } \\
\text { Wu et al } \\
\text { (2013) }\end{array}$ & $81.33 \%$ & 0.13 & 0.874 & 0.777 \\
\hline
\end{tabular}

The proposed method is compared with the existing method by Yungfeng $\mathrm{Wu}$ et al. [7]. In this method the features of VAG signal such as variance of mean squared values and form factor are extracted along with kernel density estimation method. The extracted features are directly fed to SVM classifier without feature selection which reduces the accuracy and specificity of the classifier. From the above results, it can be said that the performance of the proposed method with feature selection based on Principal Component analysis test using SVM classifier is better compared to the existing method.

\section{CONCLUSION}

In this work for classifying the knee joint VAG signals into normal and abnormal VAG signals, the input signal is decomposed into 11 sub bands using wavelet decomposition. The main signal and sub bands from D1-D3 obtained after decomposition are used as inputs for feature extraction. A large number of features such as Approximate Entropy (ApEn), Sample entropy (SaEn) and wavelet energy for main signal and each of the sub bands are extracted. The features are then fed to the SVM classifier which is trained with 38 sets of extracted features and an accuracy of $78.3 \%$ has been achieved. Also feature selection using Principal Component analysis (PCA) test is performed. With feature selection using PCA test only 6 features are chosen for training the classifiers. With feature selection the SVM classifier gives an accuracy of $82.6 \%$. The results of classification of VAG signals suggest that the parameters obtained after feature extraction using approximate entropy, sample entropy and wavelet energy provide a good discrimination between the abnormal and normal VAG signals. Moreover, the features selected by PCA test also increased the accuracy of the classifier. In this study, it is understood that compared to the other methods of feature selection PCA test is the simplest method. In case of the classifiers it is understood that SVM classifier can provide a better accuracy along with feature selection.

\section{REFERENCES}

[1]. McCoy G., McCrea J., Beverland D., Kernohan W., Mollan R., (1987) 'Vibration arthrography as a diagnostic aid in diseases of the knee'. A preliminary report, J. Bone Jt. Surg. Br. Vol.69B, No.2, pp.288-293.

[2]. Saif Nalband., Sreekrishna R.R., Amaline Prince A., (2016), 'Analysis of knee joint vibration signals using ensemple empirical mode decomposition', In proceedings of Twelfth International Multi-conference on Information Processing, Vol.89, pp.820-827.

[3]. Daubechies I., (1990), 'Wavelet transform, time-frequency localization and signal analysis', IEEE Trans. On Information Theory, Vol.36, No.1, pp. 2-8.

[4]. Elif Derya Ubeyil (2009), 'Combined neural network model employing wavelet coefficients for signal classification', Journal on Digital Signal Processing, Vol.19, pp.297-308

[5]. Mingyang Li., Wanzhong Chen., Tao Zhang., (2017), 'Classification of epilepsy EEG signals using DWT-based envelope analysis and neural 
network ensemble', Journal on Biomedical Signal Processing, Vol.31, No.1, pp.357-365

[6]. Kaizhi Liu., Xin Luo., Shanshan Yang., Suxian Cai., Fang Zheng., Yunfeng Wu., (2014), 'Classification of knee joint vibroarthrographic signals using $\mathrm{K}$ nearest neighbor algorithm', IEEE Trans., Vol.36, No.1, pp.273-279.

[7]. Yungfen wu., Suxian Cai., Shanshan Yang., Fang Zheng., Nin Xiang., (2013), 'Classification of knee joint vibration signals using bivariate feature distribution estimation and maximal posterior probability decision criterion', Journal on Entropy, Vol.15, pp.1375-1387.

[8]. Yungfen Wu., Pinnan Chen., Xin Luo., Hui Hang., Lifang Liao., Yuchen Yao., Meihong Wu., Rangaraj Rangayyan M., (2016), 'Quantification of knee joint VAG signal irregularity associated with patellofemoral joint cartilage pathology based on entropy and envelope amplitude measures', Journal on Computer methods and programs in Biomedicine, Vol.130, pp.1-12.

[9]. Tingting Mu., Ashoke Nandi K., Rangaraj Rangayyan M., (2008), 'Screening of knee joint VAG signals using the strict-2 surface proximal classifier and genetic algorithm', Journal on Computers and biology in Medicine, Vol.38, No.1, pp.1103-1111.

[10]. Ahmed Taher Azar., Hanna Ismail Elshazly., Aboul Ella Hassanien., Abeer Mohamed Elkorany., (2014), 'A random forest classifier for lymph diseases', Comput. Methods Progr. Biomed., Vol.113, No.2, pp.465-473.

[11]. Jasmin Kevric and Abdulhamit Subasi (2017), 'Comparison of signal decomposition methods in classification of EEG signals for motorimagery BCI system', Journal of Biomedical Signal Processing, Vol.36, No.1, pp.273-279.

[12]. Yentes J.M., Hunt N., Schmid K.K., Kaipust J.P., McGrath D., Stergiou N., (2013), 'The appropriate use of approximate entropy and sample entropy with short data sets', Ann. Biomed. Eng., Vol.41, No.2, pp.349-365.

[13]. Pincus S., (1991), 'Approximate entropy as a measure of system complexity', -In proceedings of the National Academy of Sciences Vol.88, No.6, pp.2297-2301.

[14]. Dengyong Zhang., Shanshan Wang., Feng Li., Jin Wang., Arun Kumar Sangaiah., Victor S. Sheng., Xiangling Ding., (2019), 'An ECG signal denoising approach based on wavelet energy and sub-band smoothing filter', Applied Sciences, Vol.9, 4698, doi:10.3390/app9224968.

[15]. Arosha Senanayake S.M.N., Owais Ahmed Malik., Mohammad Iskandar., Danish Zaheer., (2014), 'A knowledge based intelligent framework for anterior cruciate ligament rehabilitation monitoring', Journal on applied Soft Computing, Vol.20, No.2, pp.127-141.

[16]. Nitesh Singh Malan., Shiru Sharma., (2019), "Feature selection using regularized neighbourhood component analysis to enhance the classification performance of motor imagery signals", Computers in biology and medicine, Vol.107, pp.118-126.

[17]. Sridhar Krishnan., Rangaraj Rangayyan M., Douglas Bell G., Cyril B., (2000), 'Adaptive time-frequency analysis of knee joint VAG signals for noninvasive screening of articular parthology', IEEE Trans. On Biomedical Engineering, Vol.47, No.6, pp.243-265.

[18]. Le Hoang Son., Mumtaz Ali., Nguyen Dang., Nguyen Van Minh., (2015), 'A Neutrosophic recommender system for medical diagnosis based on algebraic neutrosophic measures', Journal of Biomedical Informatics, Vol.54, No.2, pp. 220-229.

[19]. Xie S., Krishnan S., (2013), 'Wavelet-based sparse functional linear model with applications to EEGs seizure detection and epilepsy diagnosis', Med. Biol. Eng. Comput. Vol.51, No.10 pp.49-60. 
[20]. Parvinnia E., Sabeti M., Zolghadri Jahromi M., Boostani R., (2014), 'Classification of EEG signals using adaptive weighted distance nearest neighbor algorithm', Journal of Computer and Information Sciences, Vol.26, pp.1-6.

[21]. Erick Odhiambo Omuya., George Onyango Okeyo., Michael Waema Kimwele., (2021), "Feature selection for classification using principal component analysis and information gain", Expert systems with applications, Vol.174, 114765.

[22]. Karthikeyan Umapathy and Sridhar Krishnan (2006), 'Modified local discriminant algorithm and its applications in analysis of human knee joint vibration signals', IEEE Trans. Biomed Eng., Vol.53, No.3, pp.517-523.

[23]. Saif Nalband., Aditya Sundar., Amalin Prince A., Anita Agarwal., (2016), 'Feature selection and classification for the detection of knee joint disorders", Computer methods and programs in biomedicine, Vol.16, 1169-2607.

[24]. Keo Sik Kim., Jeong Hwan Seo., Jin U. Kang., Chul Gyn Song., (2009), 'An enhanced algorithm for knee joint sound classification using feature extraction based on timefrequency analysis', Comput. Methods Progr. Biomed. Vol.94, No.2, pp.198-206.

[25]. Shanshan Yang., suxian Cai., Fang Zheng., Kaizhi Liu., Meihong Wu., Quan Zou., Jian Chen., (2014), 'Representation of fluctuation features in pathological knee joint vibroarthrographic signals using kernel density modeling method', Med. Eng. Phys. Vol.36, No.9, pp.1305-1311.

\section{Cite this article as :}

Alphonsa Salu S. J., Jeraldin Auxillia D, "Detection of Knee Joint Disorders using SVM Classifier", International Journal of Scientific Research in Science and Technology (IJSRST), Online ISSN : 2395-602X, Print ISSN : 2395-6011, Volume 8 Issue 5, pp. 261-271, September-October 2021. Available at doi : https://doi.org/10.32628/IJSRST218535 Journal URL : https://ijsrst.com/IJSRST218535 